\title{
VMAT for prostate cancer with 6-MV and 10-MV photons: Impact of beam energy on treatment plan quality and model-based secondary cancer risk estimates
}

\author{
MICHALIS MAZONAKIS $^{1}$, STEFANOS KACHRIS ${ }^{2}$ and JOHN DAMILAKIS ${ }^{1}$ \\ ${ }^{1}$ Department of Medical Physics, Faculty of Medicine, University of Crete, 71003 Iraklion; \\ ${ }^{2}$ Department of Radiotherapy and Oncology, University Hospital of Iraklion, 71110 Iraklion, Greece
}

Received June 29, 2020; Accepted November 20, 2020

DOI: $10.3892 / \mathrm{mco} .2021 .2251$

\begin{abstract}
The aim of the present study was to examine the effect of the photon beam energy on the volumetric modulated arc therapy (VMAT) plan quality for prostate cancer and on the risk of secondary carcinogenesis. Separate VMAT plans with 6-MV and 10-MV photons were created for 11 low-risk patients with prostate cancer. The prescribed tumor dose was 70 Gy delivered in 28 fractions. The normal tissue integral dose and parameters associated with planning target volume and organs at risk were determined by the treatment planning data. A non-linear mechanistic model considering the effects of tumor dose fractionation and cell proliferation was employed for estimating the patient-specific lifetime attributable risk (LAR) for bladder and rectal cancer induction. Data from differential dose-volume histograms were used for these risk assessments. The mean values of the planning parameters from 6-MV treatment plans differed by $0.2-3.4 \%$ from those associated with irradiation using 10-MV photons. The LAR range for developing secondary bladder malignancies varied between 0.041 and $0.129 \%$ by the patient under investigation and the beam energy used. The corresponding range for the appearance of rectal malignant diseases was 0.047-0.153\%. The mean percentage difference between the bladder cancer risks from VMAT with 6-MV and 10-MV photons was $2.6 \pm 2.3 \%$. The corresponding difference for secondary rectal malignancies was $0.7 \pm 0.6 \%$. Therefore, VMAT for prostate cancer with both 6-MV and 10-MV photons leads to clinically equivalent treatment plans and to similar secondary bladder and rectal cancer risks.
\end{abstract}

Correspondence to: $\mathrm{Dr}$ Michalis Mazonakis, Department of Medical Physics, Faculty of Medicine, University of Crete, P.O. Box 2208, 71003 Iraklion, Greece

E-mail: mazonak@uoc.gr

Key words: prostate cancer, volumetric modulated arc therapy, beam energy, treatment planning, secondary carcinogenesis

\section{Introduction}

Prostate cancer is expected to account for $21 \%$ of total new malignancies developing among men in USA during 2020 (1). Several factors associated with increasing age, ethnicity, family history, genetic and hormonal influences, increase the probability of being diagnosed with carcinoma of the prostate gland (2). The presence of this malignancy can reduce the life expectancy and also compromise the quality of life of the patients due to sexual dysfunction, urinary incontinence and bowel problems. The improvements in early disease detection and treatment have reduced the mortality rate for prostate carcinoma by $52 \%$ since 1993 and have achieved a 5-year survival rate for all-stage disease of $98 \%$ (1). External-beam radiotherapy is extensively applied for the effective management of prostate cancer (2). At present, prostate irradiation is usually performed with the modern techniques of intensity-modulated radiation therapy (IMRT) and volumetric modulated arc therapy (VMAT). These modern approaches enable the delivery of high cumulative radiation doses to the tumor site using high-energy X-ray beams generated by a linear accelerator. Both IMRT and VMAT improve the quality of the patient's treatment plan and the sparing of the adjacent normal structures compared to conventional irradiation $(3,4)$. A meta-analysis comparing the two aforementioned modulated techniques revealed that VMAT may be considered as the preferred approach to prostate cancer treatment due to its superior delivery efficiency (5).

VMAT is usually delivered with 6-MV photons in most radiation oncology centers (6). The use of 10-MV X-rays for arc therapy of prostate carcinoma has also been proposed (7-10). Pasler et al (7) demonstrated that the effect of beam energy on the target coverage and organ at risk (OAR) sparing is not significant. Different results were reported by other studies (8-10). Kleiner and Podgorsak (8) found that the use of 10-MV instead of 6-MV X-rays was associated with better conformity and sparing of the critical organs. Stanley et al (9) observed a faster dose fall-off with 10-MV photon beams. Mattes et al (10) also reported that the increase of photon beam energy resulted in dosimetric benefits. However, none of those studies discussed the issue of radiation-induced carcinogenesis due to the heavy irradiation of surrounding tissues. 
The purpose of the present study was to examine the effect of 6-MV and 10-MV photon beam energies on the VMAT plan quality for prostate cancer, as well as on the relevant risk of secondary cancer induction.

\section{Materials and methods}

Prostate cancer patients. A total of 11 consecutive patients with newly diagnosed low-risk prostate cancer, who underwent external-beam radiation therapy at the Department of Radiotherapy and Oncology of the University Hospital of Iraklion between July and December 2019, were studied. All patients had ultrasound-guided transrectal biopsy-proven clinical T1-T2aN0M0 disease with Gleason score 3+3/grade 1 and prostate-specific antigen $<10 \mathrm{ng} / \mathrm{ml}$. None of the participants had been subjected to transurethral resection and/or hormone therapy prior to irradiation. Patients with hip implants were excluded from the study. The patients had been subjected to a planning computed tomography (CT) examination with a comfortably full urinary bladder and an empty rectum. The age of each study participant is presented in Fig. 1. The mean patient's age \pm one standard deviation (SD) was $68.0 \pm 2.5$ years.

Contouring and treatment planning. The treatment planning process was carried out with the Monaco system, version 5.11.03 (Elekta Instrument AB). The CT images of the study participants were transferred to the aforementioned system. The rectum, urinary bladder, and right and left femoral heads were manually delineated and were considered as the OARs. A radiation oncologist was responsible for the contouring of the structures of interest on CT scans. The rectal boundaries were drawn from the anus to the rectosigmoid flexure. The clinical target volume (CTV) coincided with the manually delineated prostate gland. The planning target volume (PTV) was calculated as the CTV with a margin of $0.5-0.8 \mathrm{~cm}$ in all directions, except posteriorly, where a margin of $0.4 \mathrm{~cm}$ was applied (11). Moderate hypofractionated irradiation was used for the treatment of low-risk prostate cancer patients, as suggested in the literature $(11,12)$. All patients were prescribed to receive $70 \mathrm{~Gy}$ to the PTV in 28 fractions using VMAT on a newly installed medical linear accelerator (Elekta Instrument AB) emitting 6-MV and 10-MV photons.

For each study participant, two VMAT plans with 6-MV and 10-MV X-rays were generated. The applied VMAT technique consisted of two full arcs with the same isocenter in clockwise and counterclockwise directions. The beam delivery was continuous over each arc. The beam was modulated by dynamic multileaf collimation, variable dose rate and speed of gantry rotation. The dose calculations of the VMAT plans were made using a Monte Carlo algorithm. The dose constraints for the PTV and OARs were based on previous reports $(11,13)$ and they are presented in Table I. Cumulative dose-volume histograms (DVHs) of the aforementioned structures were employed to determine the relevant $\mathrm{V}_{\mathrm{i}}$, defined as the percentage of the target or OAR volume absorbing a radiation dose equal to $\mathrm{i} G y$. The normal tissue integral dose (NTID) was also calculated as the product of the average dose to a region, including normal tissues minus PTV, and the volume of this region. The number of monitor units (MU) was recorded for each plan.
Radiation-induced bladder and rectal cancer risks. Radiotherapy for prostate cancer may increase the risk of development of radiation-induced malignancies to the adjacent bladder and rectum (14). These secondary cancer risks were estimated in the present study. The DVHs of rectum and bladder derived from each VMAT plan demonstrated that these organs receive an inhomogeneous dose distribution. Parts of these OARs are exposed to primary radiation and, therefore, they receive high doses, similar to the dose delivered to the target. For radiation doses up to $\sim 2 \mathrm{~Gy}$, the risk of radiation carcinogenesis is linearly related to the absorbed dose (15). The extrapolation of the linear-no-threshold model to high therapeutic doses is currently in dispute $(15,16)$. Schneider et al (17) previously introduced the concept of the organ equivalent dose (OED), which considers the inhomogeneous dose distribution of partially in-field organs from radiotherapy. The non-linear mechanistic model is based on the use of the OED. The model parameters were defined by data obtained from Japanese A-bomb and Hodgkin cohorts for doses similar to radiation therapy (17).

Differential DVHs were employed to compute the OED of bladder and rectum from all VMAT plans with 6-MV or 10-MV photons with the formula:

$$
O E D=\frac{1}{V_{o}} \sum_{i} V_{D_{i}} \frac{e^{-a_{i}^{\prime} D_{i}}}{a_{i}^{\prime} R}\left[1-2 R+R^{2} e^{a_{i}^{\prime} D_{i}}-(1-R)^{2} e^{-\frac{a_{i}^{\prime} R}{1-R} D_{i}}\right]
$$

where $\mathrm{V}_{\mathrm{o}}$ is the overall organ volume as measured from CT scans, $\mathrm{V}_{\mathrm{Di}}$ is the organ volume receiving a radiation dose of $\mathrm{Di}$, and $\mathrm{R}$ is the organ-dependent repopulation parameter. The cell-kill parameter was calculated as follows:

$$
a_{i}^{\prime}=a+\beta \frac{1}{n} D_{i}
$$

where $\alpha$ and $\beta$ are the linear quadratic model factors and $n$ is the number of fractions delivered during the whole radiotherapy course. The excess absolute risk (EAR) for developing bladder or rectal malignancies due to VMAT for prostate cancer was estimated using the following equation:

$$
E A R=\beta_{E A R} O E D e^{\left[\gamma_{e}\left(a g e_{e}-30\right)+\gamma_{a} \ln \left(\frac{a g e_{a}}{70}\right)\right]}
$$

where $\beta_{E A R}$ is the slope of the dose-response curve in the low-dose region for individuals in Western countries, age $e_{e}$ is the patient's age at the time of irradiation, $a g e_{a}$ is the attained age of the patient and $\gamma_{e}, \gamma_{a}$ are the age-modifying factors (17). The parameters $R, \alpha, \beta, \beta_{E A R}, \gamma_{e}$ and $\gamma_{a}$ for the bladder and rectum were derived from the literature $(17,18)$ and they are summarized in Table II. The lifetime attributable risk (LAR) was calculated by summing the EAR values over an attained age from a latent period of cancer induction of 5 years after radiotherapy to a final attained age of 80 years. The LAR was calculated using the formula:

$$
L A R=\sum_{a g e_{e}+5}^{80} E A R\left(\text { age }_{e}, a e_{a}\right) \frac{S\left(a g e_{a}\right)}{S\left(a g e_{e}\right)}
$$

where the quantity $S\left(a g e_{a}\right) / S\left(a g e_{e}\right)$ refers to the probability of a male patient to survive from age $e_{e}$ to $a g e_{a}$ according to the most recent United States life tables (19). 


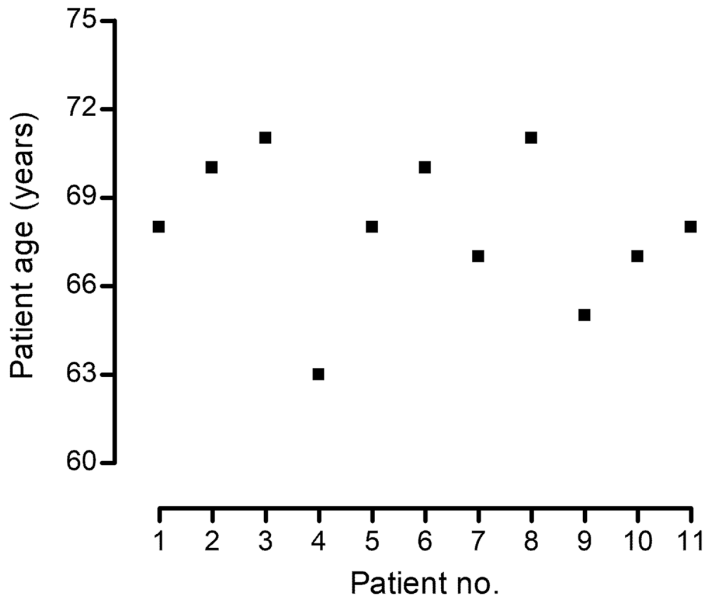

Figure 1. Age of patients with prostate cancer.

Bland-Altman analysis. The agreement of the lifetime risks for developing bladder or rectal malignancies due to VMAT plans with 6-MV photons with those from arc therapy based on the use of 10-MV X-rays was assessed using Bland-Altman analysis. This statistical test is widely used for the determination of the exact levels of agreement along with the respective confidence intervals between the two experimental methods. Bland-Altman analysis was made using the software package GraphPad Prism v.4.0 (GraphPad Software, Inc.). For each patient, the percentage difference between the organ-specific LARs estimated with the low and high photon energy was plotted against the mean LAR value. The mean percentage difference associated with bladder or rectal cancer risk was calculated. The $95 \%$ limits of agreement are presented as the mean difference $\pm 1.96 \mathrm{SD}$ of the differences.

\section{Results}

Parameters derived from VMAT plans. The mean values of the parameters associated with the target site and critical organs, as derived from the treatment plans of all patients, are summarized in Table III. The analysis of the DVHs revealed that the femoral heads were not exposed to doses up to $45 \mathrm{~Gy}\left(\mathrm{~V}_{45}=0 \%\right)$ irrespective of the $\mathrm{X}$-ray beam energy used. Moreover, no volume of the bladder or rectum received a radiation dose $>74 \mathrm{~Gy}$ for all VMAT plans $\left(\mathrm{V}_{74}=0 \%\right)$. The difference between the mean $\mathrm{V}_{\mathrm{i}}$ of the parameters of the urinary bladder and rectum, as determined by the VMAT plans with 6-MV and 10-MV photons, varied between 1.4 and $3.4 \%$. The corresponding difference for the mean $\mathrm{V}_{70}$ of the PTV and the mean NTID was found to be 0.2 and $2.7 \%$, respectively. Prostate irradiation with the high photon energy resulted in a mean MU reduction of $11.8 \%$ compared to arc therapy using low-energy X-rays.

Radiation-induced bladder and rectal cancer risks. The mean OED of bladder and rectum from the 6-MV VMAT plans of all patients was $0.65 \pm 0.18$ and $8.69 \pm 0.48 \mathrm{~Gy}$, respectively. The corresponding OED due to treatment with a higher photon energy was $0.63 \pm 0.16$ and $8.63 \pm 0.50 \mathrm{~Gy}$. The LAR for bladder cancer induction from VMAT with 6-MV photons varied between 0.042 and $0.129 \%$, whereas the use of $10-\mathrm{MV}$
Table I. Dose constraints for PTV and organs at risk.

\begin{tabular}{lc}
\hline Structure & Constraint \\
\hline PTV & $\mathrm{V}_{70} \geq 98 \%$ \\
Bladder & $\mathrm{D}_{\max } \leq 74.9 \mathrm{~Gy}$ \\
& $\mathrm{~V}_{74} \leq 25 \%$ \\
& $\mathrm{~V}_{69} \leq 35 \%$ \\
Rectum & $\mathrm{V}_{64} \leq 50 \%$ \\
& $\mathrm{~V}_{74} \leq 15 \%$ \\
& $\mathrm{~V}_{69} \leq 20 \%$ \\
& $\mathrm{~V}_{64} \leq 25 \%$ \\
Femoral heads & $\mathrm{V}_{59} \leq 35 \%$ \\
\hline
\end{tabular}

PTV, planning target volume.

Table II. Organ-specific risk parameters of the mechanistic model.

\begin{tabular}{lcc}
\hline Parameters & Bladder & Rectum \\
\hline $\mathrm{R}$ & 0.06 & 0.56 \\
$\alpha\left(\mathrm{Gy}^{-1}\right)$ & 0.219 & 0.033 \\
$\alpha / \beta(\mathrm{Gy})$ & 3.0 & 3.0 \\
$\gamma_{\mathrm{e}}$ & -0.024 & -0.056 \\
$\gamma_{\mathrm{a}}$ & 2.38 & 6.9 \\
$\beta_{\mathrm{EAR}}\left(/ 10^{4} \mathrm{PY}\right.$ Gy $)$ & 3.8 & 0.73 \\
\hline
\end{tabular}

$\mathrm{R}$, repopulation factor; $\alpha / \beta$, linear quadratic parameters; $\gamma_{\mathrm{e}}$ and $\gamma_{\mathrm{a}}$ age-modifying factors; $\beta_{\mathrm{EAR}}$ slope of the dose-response curve at low doses.

X-rays led to LARs of $0.041-0.123 \%$ (Fig. 2). The LAR range for developing secondary rectal malignancies due to VMAT with the low and high photon energy was $0.048-0.153$ and $0.047-0.150 \%$, respectively (Fig. 3).

Based on the Bland-Altman analysis, the mean percentage difference of the probability for developing bladder malignancies from VMAT plans created with 6-MV and $10-\mathrm{MV}$ photons was $2.6 \pm 2.3 \%$ (Fig. 4). The $95 \%$ limits of agreement were equal to-1.9 and $7.1 \%$ (Fig. 4). The corresponding mean difference for the second rectal cancer risk was found to be $0.7 \pm 0.6 \%$, with limits of agreement of -0.5 and $1.9 \%$ (Fig. 5).

\section{Discussion}

In the present study, the effect of 6-MV and 10-MV photons on the VMAT plans for prostate cancer and on the probability for developing secondary bladder or rectal malignancies were investigated. No attempts were made to use higher photon beam energies for treatment planning. It is well known that there is no neutron contamination when medical linear accelerators operate at 6-MV. The neutron production is also minimal for treatment with $10-\mathrm{MV}$ 
Table III. Mean value of each planning parameter \pm one SD calculated from VMAT plans with 6-MV and 10-MV photons.

\begin{tabular}{lcc}
\hline & \multicolumn{2}{c}{ Mean parameter value $( \pm \mathrm{SD})$} \\
\cline { 2 - 3 } Parameters & 6-MV VMAT & 10-MV VMAT \\
\hline PTV & & \\
$\mathrm{V}_{70}(\%)$ & & $98.8 \pm 0.3$ \\
Bladder & 0.0 & \\
$\mathrm{~V}_{74}(\%)$ & 0.0 \\
$\mathrm{~V}_{69}(\%)$ & $13.9 \pm 6.9$ & $14.3 \pm 5.1$ \\
$\mathrm{~V}_{64}(\%)$ & & \\
Rectum & 0.0 & 0.0 \\
$\mathrm{~V}_{74}(\%)$ & $6.9 \pm 2.5$ & $7.0 \pm 2.3$ \\
$\mathrm{~V}_{69}(\%)$ & $12.8 \pm 4.3$ & $13.2 \pm 4.3$ \\
$\mathrm{~V}_{64}(\%)$ & $17.4 \pm 5.5$ & \\
$\mathrm{~V}_{59}(\%)$ & & 0.0 \\
Femoral heads & 0.0 & $537.6 \pm 34.4$ \\
$\mathrm{~V}_{45}(\%)$ & & $122.7 \pm 17.7$ \\
Delivery time & $605.2 \pm 43.2$ & \\
Monitor units & & \\
Normal tissues & $126.1 \pm 18.5$ & \\
NTID (Gy/l) &
\end{tabular}

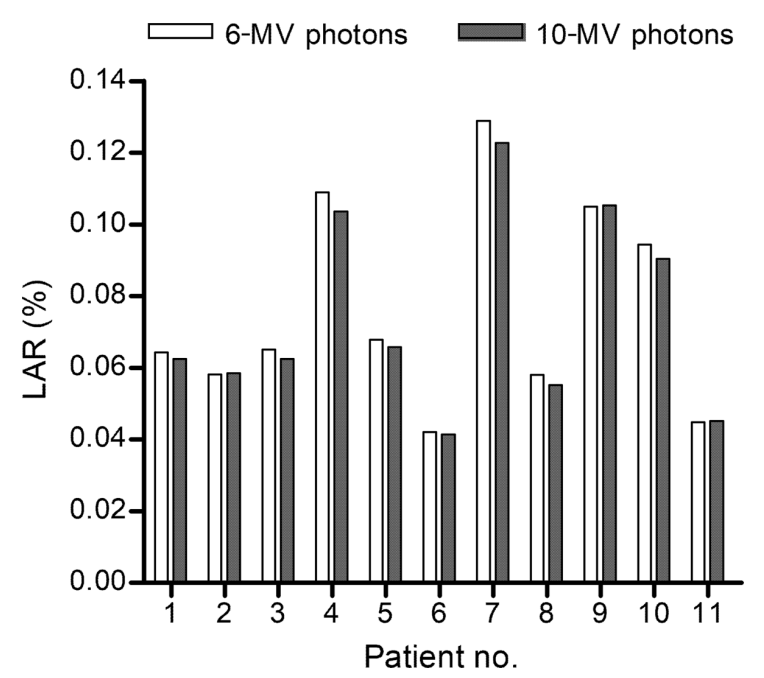

Figure 2. LAR for the appearance of second bladder cancer due to VMAT of prostate cancer with 6-MV and 10-MV photons. VMAT, volumetric modulated arc therapy; LAR, lifetime attributable risk.

X-rays (20). By contrast, the contribution of the neutron dose to the total dose of critical organs becomes significant when radiation therapy is delivered with $15-\mathrm{MV}$ or $18 \mathrm{MV}$ photon beams (20).

The DVH parameters of the two plans of each patient satisfied the previously published dose constraints $(11,13)$. The differences in the mean values of the parameters related

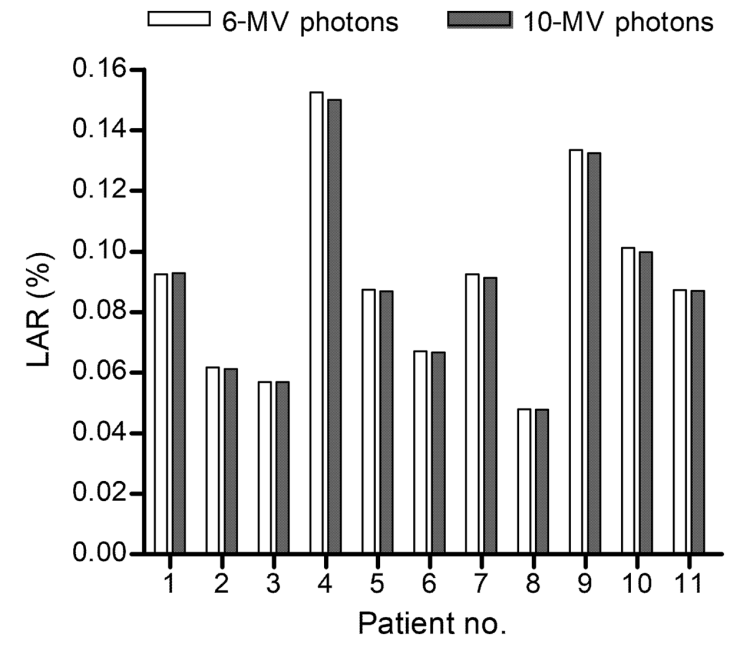

Figure 3. LAR for the appearance of second rectal cancer due to VMAT of prostate cancer with 6-MV and 10-MV photons. VMAT, volumetric modulated arc therapy; LAR, lifetime attributable risk.

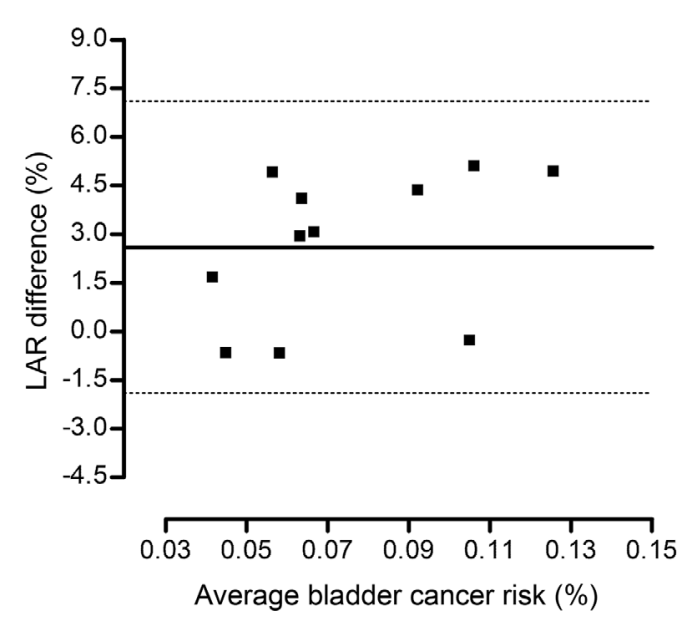

Figure 4. Bland-Altman plot showing the difference between the LAR estimates for second bladder cancer induction due to VMAT of prostate carcinoma with 6-MV and 10-MV photons vs. the mean LAR. Solid line, mean risk difference; dotted lines, $95 \%$ limits of agreement. VMAT, volumetric modulated arc therapy; LAR, lifetime attributable risk.

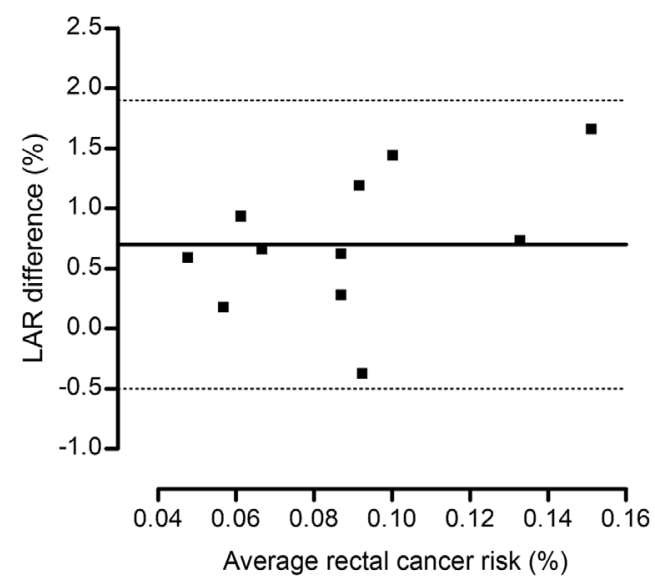

Figure 5. Bland-Altman plot showing the difference between the LAR estimates for second rectal cancer induction due to VMAT for prostate carcinoma with 6-MV and 10-MV photons vs. the mean LAR. Solid line, mean risk difference; dotted lines, $95 \%$ limits of agreement. VMAT, volumetric modulated arc therapy; LAR, lifetime attributable risk. 
to PTV, OARs and surrounding normal tissues, as defined by the plans with 6-MV and 10-MV photon energies, were found to be rather small. The aforementioned minor discrepancies are consistent with the results of previous reports on IMRT (21) and VMAT (7) for prostate cancer. In the present study, the only noteworthy discrepancy between the plans generated with the two different photon energies was observed for the treatment delivery time. The mean value of the MUs calculated for 10-MV VMAT plans was by $11.8 \%$ lower compared with that associated with $6-\mathrm{MV}$ treatment.

The lifetime risk for radiotherapy-induced bladder malignancies varied between 0.041 and $0.129 \%$ by the patient under investigation and the photon energy used for VMAT of prostate cancer. The corresponding probability for developing secondary rectal malignancies was $0.047-0.153 \%$. The lifetime risks of carcinogenesis were estimated from a patient group with a mean age of 68 years subjected to hypofractionated VMAT for prostate carcinoma up to a dose of $70 \mathrm{~Gy}$. Limited information has been published on the probability of developing secondary malignancies from prostate irradiation using hypofractionation schedules $(13,22)$. The cancer risks of the present study are consistent with those of a previous report on hypofractionated treatment with 6-MV photons at the ages of 60 and 70 years (13), reporting lifetime bladder and rectal cancer risks of $0.06-0.18 \%$ and $0.07-0.20 \%$, respectively. Stokkevag et al (22) provided a wide range of bladder and rectal cancer risks of $0.01-0.80 \%$ for 60 -year-old patients receiving $67.5 \mathrm{~Gy}$ to the prostate and simultaneously $60 \mathrm{~Gy}$ to the seminal vesicles in 25 fractions with 6-MV VMAT. Their estimates were obtained with a linear-plateau association and a bell-shaped competition model. Bladder and rectal cancer risks from standard fractionated IMRT and VMAT for prostate carcinoma have also been reported $(18,23,24)$. These theoretical risks were estimated for total tumor doses of 75.6-78.0 Gy in daily fractions of 1.8-2.0 Gy. Murray et al (18) and Fontenot et al (23) did not report lifetime risks of carcinogenesis. Sanchez-Nietto et al (24) reported lifetime probabilities of $0.4-0.5 \%$ from IMRT and VMAT for a prostate cancer patient aged 60 years.

The Bland-Altman statistical test revealed that the bladder cancer risk associated with arc therapy using the low photon energy may be up to $7.1 \%$ higher or $1.9 \%$ lower than the respective risk value from irradiation with the high energy of $\mathrm{X}$-rays in $95 \%$ of the cases. The $95 \%$ limits of agreement for the rectal cancer risk were -0.5 and $1.9 \%$. These narrow limits clearly indicate that the differences between the VMAT plans created with 6-MV and 10-MV photons in the assessment of the second cancer risks are minor.

The cancer risk assessments in the present study were carried out for the bladder and rectum, which are partly exposed to primary radiation during VMAT for prostate cancer. The use of data from treatment planning systems for out-of-field organ dose calculations is not recommended (25). Different approaches, based either on direct measurements using physical phantoms $(26,27)$ or on Monte Carlo simulations (28), may be applied for assessing the out-of-field organ doses and the relevant probabilities of carcinogenesis. The relatively small sample of patients with low-risk prostate carcinoma should be considered as a limitation of the present study. It should be noted that this study provided lifetime bladder and rectal cancer risk estimates derived from the use of a non-linear mechanistic model introduced by Schneider et al (17). The model-based risk predictions may contain several uncertainties. These uncertainties are associated with the definition of the organ-specific parameters of the mechanistic model. The use of the absolute values of the model-based cancer risk predictions in clinical practice must be viewed with caution. The PTV of the study participants included only the prostate gland. Further studies are required to examine the effect of beam energy on the probability of carcinogenesis in prostate cancer patients irradiated at sites encompassing the seminal vesicles and/or pelvic lymph nodes $(7,29)$.

In conclusion, the VMAT plans for low-risk prostate cancer patients generated with 6-MV or 10-MV photons were clinically equivalent in respect to target volume coverage and normal tissue sparing. The differences between the probabilities for developing secondary bladder and rectal malignancies due to pelvic VMAT with the low and high energy X-rays were found to be minimal. Therefore, the selection of the 10-MV photons may be considered as the optimal choice for prostate cancer treatment due to the reduction of the treatment time.

\section{Acknowledgements}

Not applicable.

\section{Funding}

No funding was received.

\section{Availability of data and materials}

The datasets used and/or analyzed during the present study are available from the corresponding author on reasonable request.

\section{Authors' contributions}

MM and JD designed the study. SK performed the target and OAR contouring on CT scans. MM and JD were responsible for treatment planning process and second cancer risk assessments. All the authors have read and approved the final version of the manuscript.

\section{Ethics approval and consent to participate}

The present study was approved by the Ethics Committee of the University Hospital of Iraklion.

\section{Patient consent for publication}

Not applicable.

\section{Competing interests}

All the authors declare that they have no competing interests. 


\section{References}

1. Siegel RA, Miller KD and Jemal A: Cancer statistics, 2020. CA Cancer J Clin 70: 7-30, 2020.

2. Perez CA, Michalski JM and Zelefsky MJ: Low-risk prostate cancer. In: Perez \& Brady's Principles and Practice of Tadiation Oncology. Halperin EC, Wazer DE, Perez CA and Brady LW (eds). Wolters Kluwer, Philadelphia, pp1560-1601, 2019.

3. Wolff D, Stieler F, Welzel G, Lorenz F, Abo-Madyan Y, Mai S, Herskind C, Polednik M, Steil V, Wenz F and Lohr F: Volumetric modulated arc therapy (VMAT) vs. Serial tomotherapy, step-and-shoot IMRT and 3D-conformal RT for treatment of prostate cancer. Radiother Oncol 93: 226-233, 2009.

4. Palma D, Vollans E, James K, Nakano S, Moiseenko V, Shaffer R, McKenzie M, Morris J and Otto K: Volumetric modulated arc therapy for delivery of prostate radiotherapy: Comparison with intensity-modulated radiotherapy and three-dimensional conformal radiotherapy. Int J Radiat Oncol Biol Phys 72 : 996-1001, 2008

5. Ren W, Sun C, Lu N, Xu Y, Han F, Liu YP and Dai J: Dosimetric comparison of intensity-modulated radiotherapy and volumetric-modulated arc radiotherapy in patients with prostate cancer: A meta-analysis. J Appl Clin Med Phys 17: 254-262, 2016

6. Cosset JM, Nassf M, Saidi R, Pugnaire J, Ben Abdennebi A and Noel A: Which photon energy for intensity-modulated radiotherapy and volumetric-modulated arc therapy in 2019? Cancer Radiother 23: 58-61, 2019.

7. Pasler M, Georg D, Wirtz H and Lutterbach J: Effect of photon-beam energy on VMAT and IMRT treatment plan quality and dosimetric accuracy for advanced prostate cancer. Strahlenther Onkol 187: 792-798, 2011.

8. Kleiner $\mathrm{H}$ and Podgorsak MB: The dosimetric significance of using 10-MV photons for volumetric modulated arc therapy for post-prostatectomy irradiation of the prostate bed. Radiol Oncol 50: 232-237, 2016.

9. Stanley DN,Popp T,Ha CS, Swanson GP,Eng TY,Papanikolaou N and Gutierez AN: Dosimetric effect of photon beam energy on volumetric modulated arc therapy treatment plan quality due to body habitus in advanced prostate cancer. Pract Radiat Oncol 5 : e625-e633, 2015

10. Mattes MD, Tai C, Lee A, Ashamalla $\mathrm{H}$ and Ikoro NC: The dosimetric effects of photon energy on the quality of prostate volumetric modulated arc therapy. Pract Radiat Oncol 4: e39-e44, 2014.

11. Lee WR, Dignam JJ, Amin MB, Bruner DW, Lo D, Swanson GP, Shah AB, D'Souza DP, Michalski JM, Dayes IS, et al: Randomized phase III noninferiority study comparing two radiotherapy fractionation schedules in patients with low-risk prostate cancer. J Clin Oncol 34: 2325-2332, 2016.

12. Morgan SC, Hoffman K, Loblaw DA, Buyyounouski MK, Patton C, Barocas D, Bentzen S, Chang M, Efstathiou J, Greany $\mathrm{P}$, et al: Hypofractionated radiation therapy for localized prostate cancer: Executive summary of an ASTRO, ASCO, and AUA evidence-based guideline. J Urol 201: 528-534, 2019.

13. Mazonakis M, Kachris S and Damilakis J: Secondary bladder and rectal cancer risk estimates following standard fractionated and moderately hypofractionated VMAT for prostate carcinoma. Med Phys 47: 2805-2813, 2020.

14. Wallis CJ, Mahar AL, Choo R, Herschom S, Kodama RT, Shah PS, Danjoux C, Narod SA and Nam RK: Second malignancies after radiotherapy for prostate cancer: Systematic review and meta-analysis. BMJ 352: i851, 2016.

15. Dasu A and Toma-Dasu I: Models for the risk of secondary cancer from radiation therapy. Phys Med 42: 232-238, 2017.

16. Mazonakis $\mathrm{M}$ and Damilakis J: Cancer risk after radiotherapy for benign diseases. Phys Med 42: 285-291, 2017.

17. Schneider U, Sumila M and Robotka J: Site-specific dose-response relationships for cancer induction from the combined Japanese A-bomb and Hodgkin cohorts for doses relevant to radiotherapy. Theor Biol Med Model 8: 27, 2011.

18. Murray LJ, Thompson CM, Lilley J, Cosgrove V, Franks K, Sebag-Montefiore D and Henry AM: Radiation-induced second primary cancer risks from modern external beam radiotherapy for early prostate cancer: Impact of stereotactic ablative radiotherapy (SABR), volumetric modulated arc therapy (VMAT) and flattening filter free (FFF) radiotherapy. Phys Med Biol 60: 1237-1257, 2015.
19. Arias E: United States life tables, 2017. Natl Vital Stat Rep 68: $1-66,2019$.

20. Kry SF, Salehpour M, Followill DS, Stovall M, Kuban DA, White RA and Rosen II: The calculated risk of fatal secondary malignancies from intensity-modulated radiation therapy. Int $\mathbf{J}$ Radiat Oncol Biol Phys 62: 1195-1203, 2005.

21. Pirzkall A, Carol MP, Pickett B, Xia P, Roach M III and Verhey LJ: The effect of beam energy and number of fields on photon-based IMRT for deep-seated targets. Int J Radiat Oncol Biol Phys 53: 434-442, 2002.

22. Stokkevag CH, Engeseth GM, Hysing LB, Ytre-Hauge KS, Ekanger $\mathrm{C}$ and Muren LP: Risk of radiation-induced secondary rectal and bladder cancer following radiotherapy of prostate cancer. Acta Oncol 54: 1317-1325, 2015.

23. Fontenot JD, Lee AK and Newhauser WD: Risk of secondary malignant neoplasms from proton therapy and intensity modulated X-ray therapy for early-stage prostate cancer. Int J Radiat Oncol Biol Phys 74: 616-622, 2009.

24. Sanchez-Nietto B, Romero-Exposito M, Terron JA, Irazola L, Garcia Hernandez MT, Mateos JC, Rosello J, Planes D, Paiusco M and Sanchez-Doblado F: External photon radiation treatment for prostate cancer: Uncomplicated and cancer-free probability assessment of 36 plans. Phys Med 66: 88-96, 2019.

25. Kry SF, Bednarz B, Howell RM, Dauer L, Followill D, Klein E, Paganetti H, Wang B, Wuu CS and George Xu X: AAPM TG-158: Measurement and calculation of doses outside the treated volume from external-beam radiation therapy. Med Phys 44: e391-e429, 2017.

26. Mazonakis M, Damilakis J, Varveris H, Theoharopoulos N and Gourtsoyiannis N: A method of estimating fetal dose during brain radiation therapy. Int J Radiat Oncol Biol Phys 44: 455-459, 1999.

27. Mazonakis M, Damilakis J, Varveris $\mathrm{H}$ and Gourtsoyiannis $\mathrm{N}$ : Therapeutic external irradiation in women of reproductive age: Risk estimation of hereditary effects. Br J Radiol 77: 847-850, 2004.

28. Bednarz B, Athar B and Xu XG: A comparative study on the risk of second primary cancers in out-of-field organs associated with radiotherapy of localized prostate carcinoma using Monte Carlo-based accelerator and patient models. Med Phys 37: 1987-1994, 2010

29. Bahtiyar N, Onaran I, Aydemir B, Batykara O, Toplan S, Agaoglu FY and Akyolcu MC: Monitoring of platelet function parameters and microRNA expression levels in patients with prostate cancer treated with volumetric modulated arc radiotherapy. Oncol Lett 16: 4745-4753, 2018. 